\title{
Intravitreal Bevacizumab for Photodynamic Therapy- Induced Massive Macular Detachment in Acute Central Serous Chorioretinopathy
}

\author{
Maria G. Kotoula Fani Zacharaki Evangelia E. Tsironi \\ Department of Ophthalmology, University of Thessaly School of Medicine, \\ Larissa, Greece
}

\section{Key Words}

Bevacizumab · Central serous chorioretinopathy · Photodynamic therapy

\begin{abstract}
We present a long followed up case of acute central serous chorioretinopathy (CSC) complicated by a severe visual loss due to massive pigment epithelium detachment of the macula after a full-dose photodynamic therapy (PDT). Rapid anatomical and functional improvement was observed after a single intravitreal injection of bevacizumab. To our knowledge, we report the first case of PDT-treated CSC complicated by severe visual loss. We can only speculate that the serous detachment of the posterior pole might have been caused by PDT-induced VEGF overexpression, explaining such an impressive response to Avastin treatment.
\end{abstract}

\section{Introduction}

During the period 2000-2005, photodynamic therapy (PDT) with verteporfin was considered a therapy of choice for certain conditions with choroidal neovascularisation (CNV). Conventional PDT has been also described as an effective and safe therapeutic option for central serous chorioretinopathy (CSC) [1].

\section{Case Report}

A 56-year-old woman presented to our emergency care unit for OD painless vision reduction and metamorphopsia lasting for 2 days. Her best corrected visual acuity (BCVA) was 0.7 OD and 1.0 OS

Dr. Maria Kotoula, MD 
General health and ocular history were unremarkable. Fundus examination and OCT3 imaging revealed a foveal neuroretinal detachment and fluorescein angiography (FA) showed two juxtafoveal points of leakage (fig. 1). The patient was put under observation. Two weeks later she presented with further vision deterioration and she stated for special vision demands. Her OD BCVA dropped to 0.4 and she agreed to be treated by PDT. Conventional dose of verteporfin infusion and laser energy parameters used in our patient were identical to those used in the TAP study [2] and the laser spot size of 3,000 $\mu \mathrm{m}$ was centered on the angiographic leakage in the FA. Only twelve hours later the patient noticed severe vision loss in the treated eye. BCVA was reduced to 0.025 and fundus examination revealed a massive macular detachment. A large serous pigment epithelium detachment was apparent on OCT. Fluorescein and indocyanine green angiography showed a dark choroidal nonperfusion area with a late hyperfluorescence in FA (fig. 2) giving an impression of occult CNV. Fifteen days after PDT the visual acuity remained unchanged. After signing an informed consent, the patient underwent an intravitreal injection of bevacizumab (Avastin) $1.25 \mathrm{mg}$ in $0.05 \mathrm{ml}$. In the following fifteen days, submacular fluid was progressively absorbed together with BCVA improvement that was 0.4 at the fifteenth day after injection, 0.8 at the first month and 0.9 at months three and six (fig. 3 ). At the last follow-up visit, almost 5 years since CSC involvement, BCVA remains stable and the integrity of the involved macular area is apparent on OCT.

\section{Discussion}

Pathophysiology of CSC remains partly unclear and treatment modalities outcomes have not concluded in a standard treatment protocol. Thermal laser photocoagulation treatment at the leaking spot has been used for decades if not too close to the foveola [1]. The rationale of using PDT for submacular fluid resolution is based on the observation that the foci of subretinal fluorescein leakage are usually accompanied by choroidal vascular hyperpermeability, this being a therapeutic target of PDT [1].

To our knowledge, we report the first case of PDT-treated CSC complicated by severe visual loss. According to the TAP study, there are few cases of PDT complicated by severe visual loss due mostly to massive subretinal hemorrhage and, rarely, to massive detachment of the posterior pole. However, these complications were reported for CNV cases only [3].

Although PDT has been reported as a safe treatment in CSC, in rare cases it can be complicated with extensive choroidal vascular damage and VEGF overexpression [4]. We believe that the immediate anatomical and visual improvement after we treated our patient with antiVEGF agent was due to the reduction of PDT-induced VEGF overexpression.

The last years, half-dose verteporfin was suggested as a precaution against permanent retinal pigment epithelium or choriocapillaris damage [5]. Recently, favourable results were also reported in patients with acute type CSC treated with halfdose verteporfin [5], the safe lowest effective dose being reported as $30 \%$ of the full dose [6]. Unfortunately it was not a usual clinical practice at the time we treated our patient. Hopefully, the reduction of the PDT dose will also protect against posttreatment severe visual loss.

\section{Disclosure Statement}

The authors have not received grant support or research funding and they do not have any proprietary interests in the materials described in the article. 


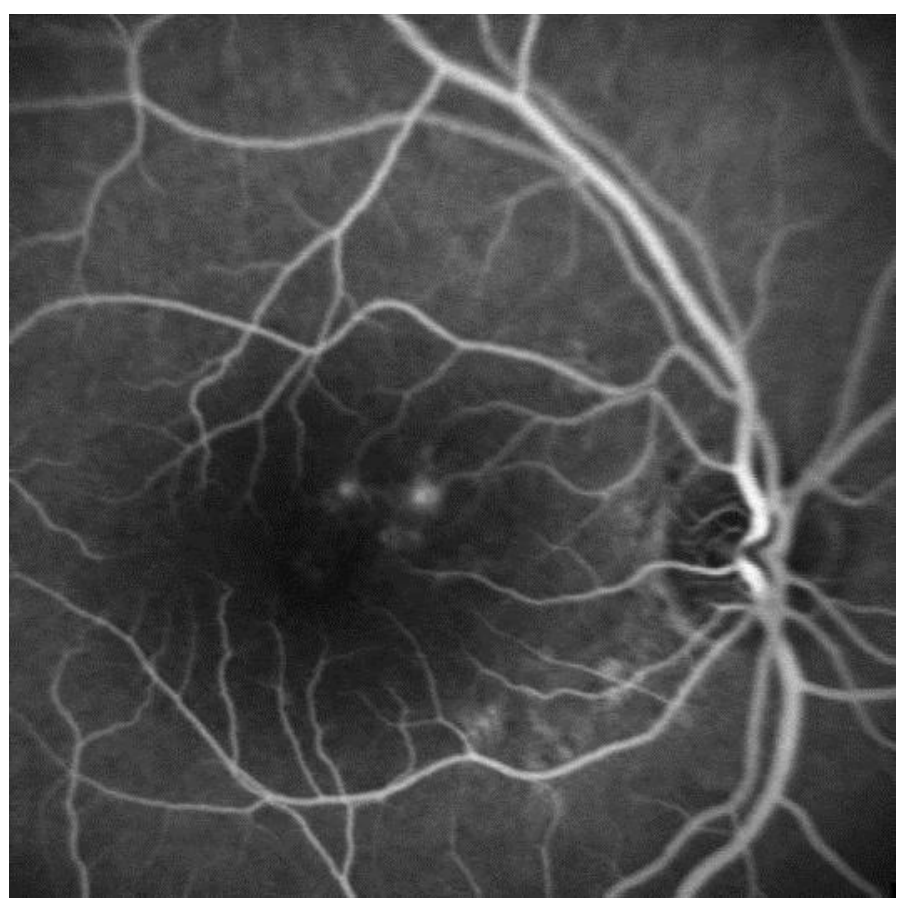

Fig. 1. Fluorescein angiography at the baseline visit revealing two juxtafoveal points of leakage. Best corrected visual acuity is 0.7 .

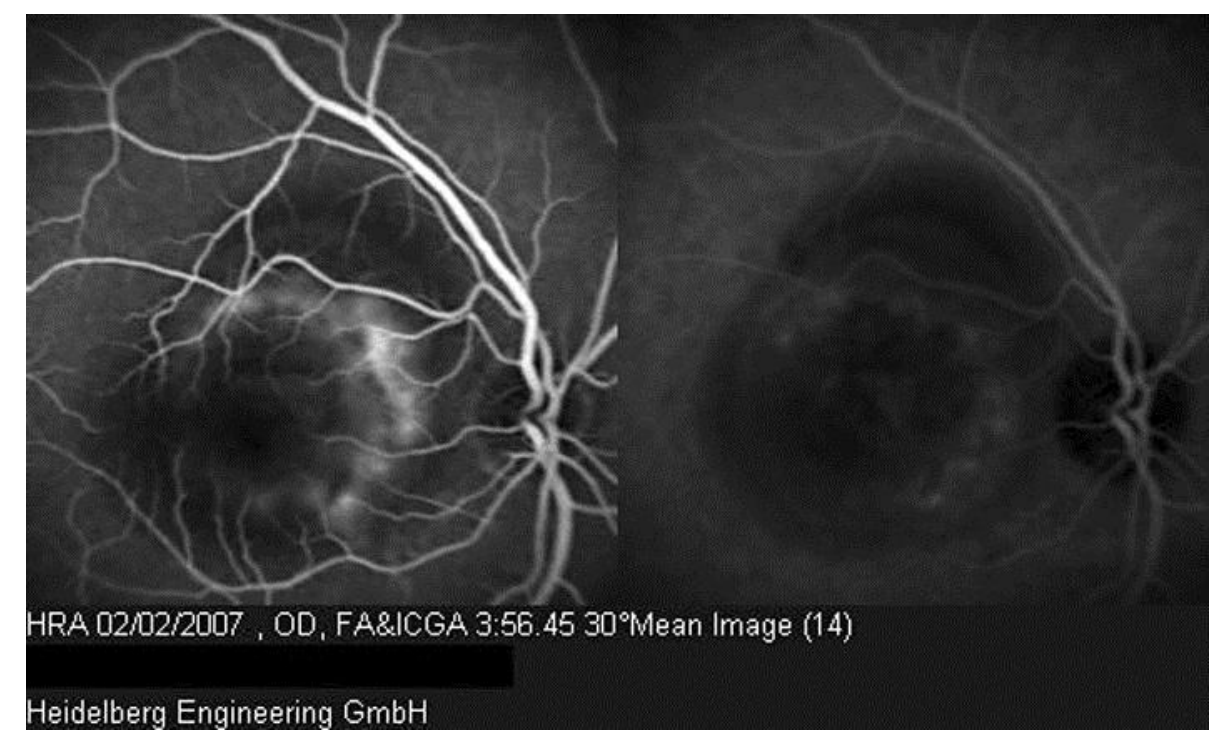

Fig. 2. Fluorescein and indocyanine green angiography at the day following the PDT session, showing a dark choroidal nonperfusion area with a late hyperfluorescence in FA due to serous pigment epithelium detachment. Best corrected visual acuity is 0.025 . 


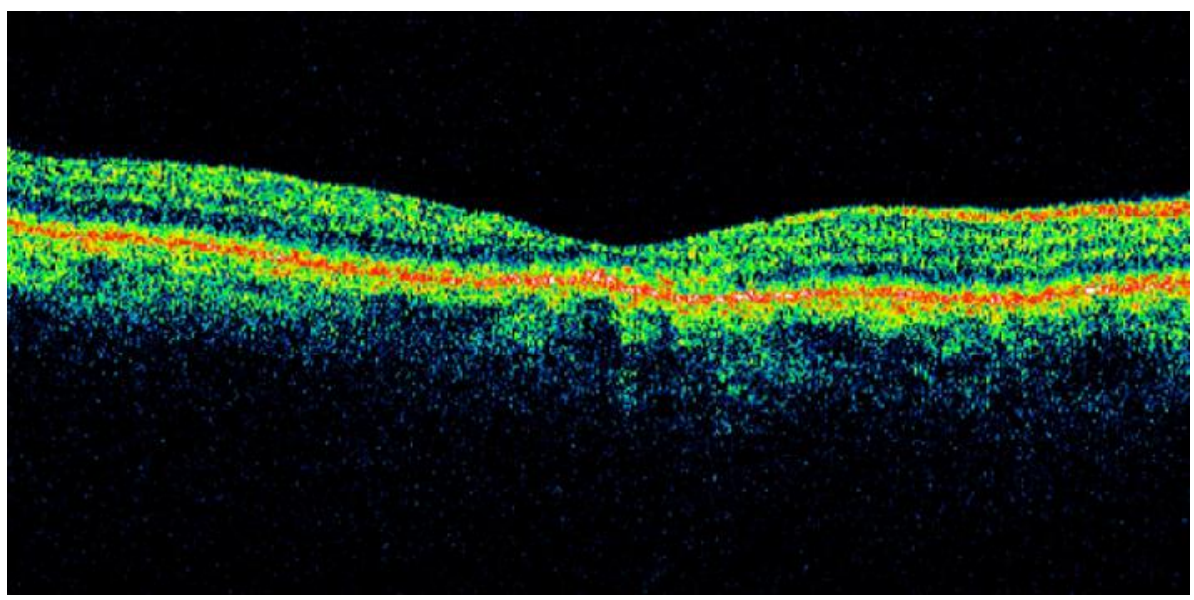

Fig. 3. OCT image 6 months after the Avastin injection, depicting a complete absorption of the fluid. Best corrected visual acuity is 0.9 .

\section{References}

1 Wang M, Munch IC, Hasler PW, Prünte C, Larsen M: Central serous chorioretinopathy. Acta Ophthalmol 2008;86:126-145

$>2$ TAP Study Group: Photodynamic therapy of subfoveal choroidal neovascularisation in age-related macular degeneration with verteporfin - TAP report 1. Arch Ophthalmol 1999;117:1329-1345.

-3 Arnold JJ, Blinder KJ, Bressler NM, et al: Acute severe visual acuity decrease after photodynamic therapy with verteporfin: case reports from randomized clinical trials - TAP and VIP Report No 3. Am J Ophthalmol 2004;137:683-696.

-4 Schmidt-Erfurth U, Schlötzer-Schrehard U, Cursiefen C, Michels S, Beckendorf A, Naumann GO: Influence of photodynamic therapy on expression of vascular en dothelial growth factor (VEGF), VEGF receptor 3 , and pigment epithelium-derived factor. Invest Ophthalmol Vis Sci 2003;44:4473-4480.

$\checkmark 5$ Chan WM, Lai TY, Lai RY, Liu DT, Lam DS: Half-dose verteporfin photodynamic therapy for acute central serous chorioretinopathy: one-year results of a randomized controlled trial. Ophthalmology 2008;115:1756-1765.

6 Zhao MW, Zhou P, Xiao HX, Lv YS, Li CA, Liu GD, Li XX: Photodynamic therapy for acute central serous chorioretinopathy: the safe effective lowest dose of verteporfin. Retina 2009;29:1155-1161. 